Artículo

\title{
Efecto del tamaño interno de la colmena en la producción de cría, miel y polen en colonias de Apis mellifera en el altiplano central de México
}

\author{
Alfonso Hernández Carlos ${ }^{a}$ \\ Ignacio Castellanos ${ }^{a *}$ \\ ${ }^{a}$ Universidad Autónoma del Estado de Hidalgo. Centro de Investigaciones Biológicas. Km \\ 4.5 carretera Pachuca-Tulancingo s/n, 42184, Hidalgo, México.
}

*Autor de correspondencia: ignacioe.castellanos@gmail.com

\section{Resumen:}

El objetivo del estudio fue analizar el efecto del tamaño interno de la colmena sobre la fortaleza (superficie con cría) y reservas de alimento (superficie con miel y polen) de abejas melíferas en la temporada invernal en una región semiárida del altiplano central mexicano. Se utilizaron cuatro bastidores de colmenas tipo Jumbo $(45 \times 28 \mathrm{~cm})$ dentro de cámaras de cría con tres tamaños internos (52.2, 42.3 y 23.9 L), que contenían el mismo número de abejas melíferas. De manera simultánea se registraron las temperaturas dentro de las colmenas para determinar si la temperatura de la cámara de cría variaba con el volumen. La colmena de mayor tamaño, que corresponde al tipo Jumbo y la más utilizada en el altiplano central mexicano, presentó los valores más bajos de fortaleza y reservas de alimento, así como la menor temperatura interna. Estos resultados muestran que la utilización de colmenas tipo Jumbo puede repercutir en una disminución de la productividad de miel y polen para los apicultores del altiplano central de México, por lo que es necesario implementar alguna práctica o mecanismo que permita mantener las colonias de abejas fuertes para la cosecha de primavera.

Palabras clave: Apis mellifera, Colmena, Cámara de cría, Invierno, Temperatura, Miel. 
Recibido: 16/08/2018

Aceptado: 09/06/2019

\section{Introducción}

El clima juega un papel fundamental en la actividad y el comportamiento de los insectos sociales $^{(1)}$. Por ejemplo, la actividad de vuelo de Apis mellifera tiene una respuesta lineal positiva con la temperatura ambiental desde los 14 hasta los $22{ }^{\circ} \mathrm{C}^{(2,3)}$ y por encima de 22 ${ }^{\circ} \mathrm{C}$, el pecoreo va disminuyendo hasta detenerse a los $35{ }^{\circ} \mathrm{C}^{(3)}$. La postura de huevos de la abeja reina de esta especie inicia a los $24{ }^{\circ} \mathrm{C}$ y alrededor de los $33{ }^{\circ} \mathrm{C}$ llega a su máxima capacidad, disminuyendo posteriormente ${ }^{(4)}$. Así mismo, la elección de las flores por la abeja melífera depende de muchos factores, pero en primer lugar de la disponibilidad floral, es decir, depende de las especies vegetales cuya floración coincide con el periodo de pecoreo y esta floración depende de las condiciones climáticas ${ }^{(5,6)}$.

Las abejas almacenan grandes cantidades de miel y polen para proporcionar energía y proteína a las crías, así como para la termorregulación, lo que permite mantener el nido de cría entre los 32 y $36{ }^{\circ} \mathrm{C}$ para el desarrollo adecuado de las larvas ${ }^{(7,8)}$, a pesar de tener temperaturas exteriores que varían entre -20 y $48{ }^{\circ} \mathrm{C}$. Para mantener estable la temperatura dentro de la colmena, las abejas emplean mecanismos activos y pasivos. Dentro de los mecanismos activos se encuentra la actividad física para generar calor (e.g., por medio de la contracción de los músculos alares) o disminuir la temperatura (e.g., empleando las alas para la ventilación $)^{(1,7,8)}$. Los mecanismos pasivos incluyen la selección del nido de cría y el desplazamiento de la cría a regiones con una temperatura más favorable ${ }^{(1,8)}$.

En general, las abejas silvestres de A. mellifera eligen un nido de cría basándose en diferentes características como el tamaño, altura y orientación de la entrada, así como su volumen interno $^{(9,10,11)}$. Por ejemplo, las obreras de $A$. mellifera seleccionan nidos de cría con volúmenes entre 15 y $100 \mathrm{~L}$, aunque el tamaño más recurrente es de $35 \mathrm{~L}^{(11)}$. Las características que las abejas seleccionan son importantes, ya que el nido de cría provee de protección contra temperaturas adversas y de estabilidad térmica para las abejas ${ }^{(8,12,13)}$. En la apicultura, el lugar y el tamaño del nido de cría es determinado por el hombre y a este espacio se le conoce como colmena ${ }^{(14)}$. En México, en términos generales, se manejan dos tipos de colmenas tecnificadas: Jumbo y Langstroth ${ }^{(15)}$. El espacio interno de la cámara de cría de estas colmenas es de 52.1 y 41.7 L respectivamente ${ }^{(16)}$.

Las colmenas tecnificadas poseen estructuras móviles que permiten aumentar el espacio para la puesta de la reina, lo que posibilita incrementar el tamaño de la colonia o 
disminuirlo cuando los factores ambientales externos son adversos ${ }^{(17)}$. Por lo tanto, el manejo adecuado del espacio puede influir en la superviviencia de las colonias en épocas críticas $^{(13,18)}$.

En años recientes se han presentado múltiples cambios en los eventos meteorológicos, propiciando temporales erráticos y heladas tempranas, lo cual no favorece las condiciones óptimas para el desarrollo de la flora apícola ${ }^{(19,20,21)}$. Esto hace necesario evaluar cómo el espacio interno de las colmenas utilizadas en México está repercutiendo en el mantenimiento de las poblaciones en la época invernal (cuando las temperaturas bajan y las abejas deben calentar el nido de cría por medios activos), y determinar el efecto que tiene el tamaño de la colmena sobre las abejas y algunos de sus productos como la miel y polen, bajo condiciones específicas de cada región apícola. Por lo tanto, el objetivo del presente trabajo fue determinar el efecto que tiene el tamaño interno de la colmena en la producción de progenie y el almacenamiento de miel y polen, así como en la temperatura interna del nido de cría, en colonias de $A$. mellifera en la temporada invernal.

\section{Material y métodos}

\section{Área de estudio}

La investigación se llevó a cabo en la localidad de Huitzila, municipio de Tizayuca, el cual

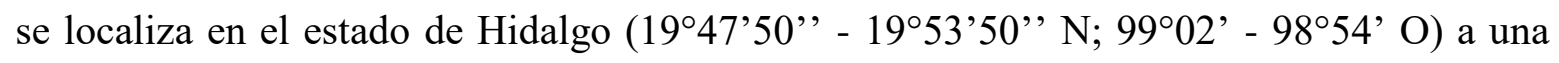
altitud de 2,260 $\mathrm{msnm}^{(22)}$ dentro del altiplano central mexicano. El clima de esta región es templado subhúmedo con lluvias en verano, temperatura media anual de $15.1{ }^{\circ} \mathrm{C}$ y precipitación promedio anual de $627 \mathrm{~mm}$. El mes más frío es enero, con una temperatura mínima y media de 1.4 y $11.5^{\circ} \mathrm{C}$, respectivamente, mientras que el mes más cálido es mayo, con una temperatura mínima y media de 8.7 y $17.8^{\circ} \mathrm{C}$, respectivamente ${ }^{(23)}$.

\section{Preparación de abejas}

En diciembre de 2016, al terminar el flujo de néctar y polen, se seleccionaron 10 colonias que presentaban desarrollo homogéneo y de éstas se extrajeron aproximadamente 120,000 abejas adultas $^{(24)}$ para el experimento, de las cuales se asignaron 10,000 obreras de forma homogénea a cada una de las 12 colonias que se utilizaron en el experimento. Para contar a las abejas, se utilizó una cámara de crecimiento a $-2{ }^{0} \mathrm{C}$ durante 10 min (Shel Lab, modelo LI15) para inmovilizarlas.

Cada uno de los 12 grupos de 10,000 abejas se colocó dentro de 12 colmenas de diferentes

volúmenes internos con capacidad para albergar diferente número de bastidores de cámara 
tipo Jumbo (45 x $28 \mathrm{~cm}$ ) (Figura 1). Se emplearon 4 colmenas con capacidad para albergar 4 bastidores, 4 para albergar 8 bastidores y 4 para 10 bastidores, cuyas medidas externas fueron de $51 \times 30 \times 21 \mathrm{~cm}$ para 4 bastidores, 51 × $30 \times 34 \mathrm{~cm}$ para 8 bastidores y 51 × $30 \times$ $41 \mathrm{~cm}$ para 10 bastidores, lo que permitió tener volúmenes internos de $23.9 \mathrm{~L}$ (colmena experimental), $42.3 \mathrm{~L}$ (colmena tipo Langstroth) y $52.2 \mathrm{~L}$ (colmena tipo Jumbo), respectivamente. Las colmenas se construyeron de madera de pino de $2 \mathrm{~cm}$ de espesor y se trataron con parafina. A cada colonia con 10,000 abejas se le colocaron 4 bastidores con cera estampada y una abeja reina recién fecundada de un mismo pie de cría y del mismo lote de una mezcla heterogénea de las razas Italiana y Carniola, lo cual permitió que las colonias comenzaran con las mismas condiciones iniciales (las reinas eran hermanas). A cada colmena se le suministraron $600 \mathrm{ml}$ de jarabe preparado con agua y azúcar en una proporción de 1:1 y 150 g de suplemento proteico (Apitir plus de la empresa Tirtécnica) cada ocho días ${ }^{(25,26)}$. El suministro de jarabe y suplemento proteico se mantuvo durante todo el experimento.

Figura 1: Colmenas con diferentes volúmenes internos utilizadas en el experimento, A) colmena exprimental, B) colmena tipo Langstroth y C) colmena tipo Jumbo

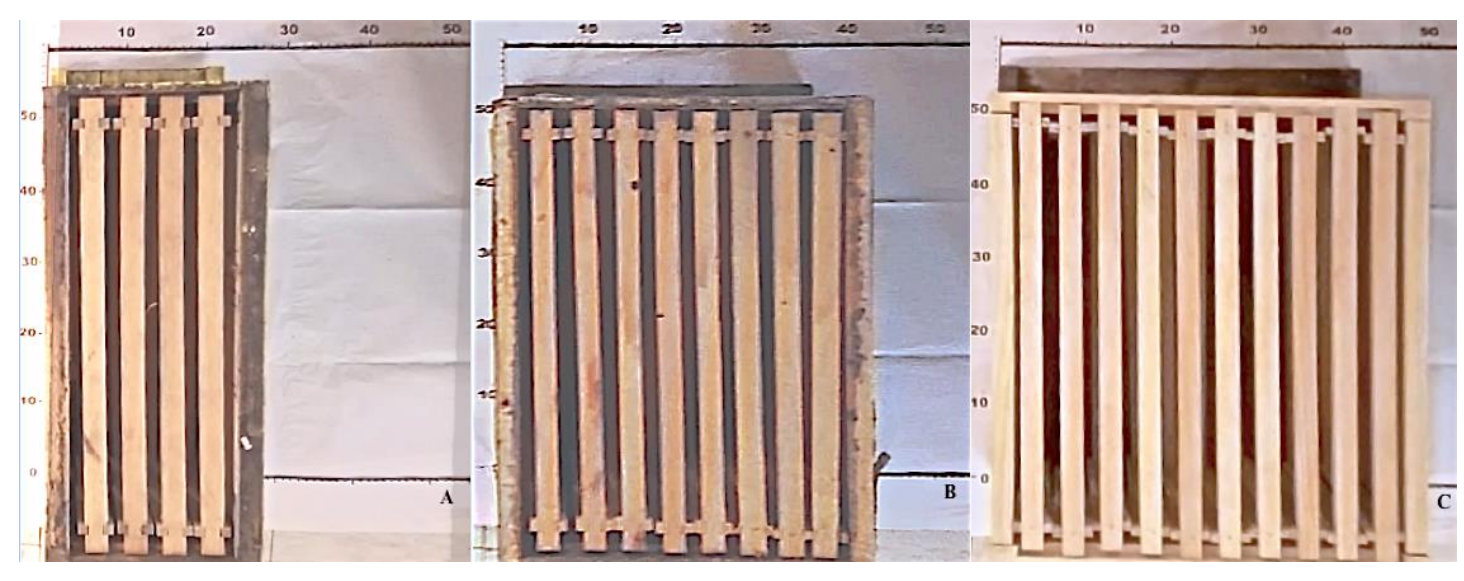

\section{Experimento}

Se utilizaron cuatro repeticiones para cada tamaño de colmena, dando un total de 12 unidades experimentales que fueron colocadas en un diseño completamente al azar, a una distancia de $2 \mathrm{~m}$ entre hileras y $1 \mathrm{~m}$ entre colmenas de la misma hilera ${ }^{(17)}$. Se registró la temperatura interna de las colmenas utilizando 24 dataloggers (Thermochron iButton modelo DS1921G). En cada colmena se colocaron dos dataloggers en el tercer bastidor, uno en el centro y el segundo a la altura del cabezal para determinar si la temperatura interna variaba con el volumen de la cámara de cría. Adicionalmente, se registró la 
temperatura ambiental a la sombra utilizando 12 dataloggers. Las temperaturas se registraron cada 60 min durante el experimento.

El experimento comenzó la primera semana de diciembre y terminó el 28 de abril, al inicio de la floración. Se contabilizó la superficie con cría, miel y polen utilizando una mica de plástico transparente cuadriculado en centímetros ${ }^{(24)}$ (Figura 2). No se contabilizó el número de individuos adultos para no romper la cohesión social ${ }^{(24)}$. Los registros de cría, miel y polen se llevaron a cabo en ambos lados de cada bastidor únicamente en tres fechas ( 2 de febrero, 7 de marzo y 28 de abril) para preservar la cohesión social ${ }^{(24)}$.

Figura 2: A) Bastidor con y B) sin la cuadrícula transparente utilizada para la cuantificación de la superficie con cría, miel y polen

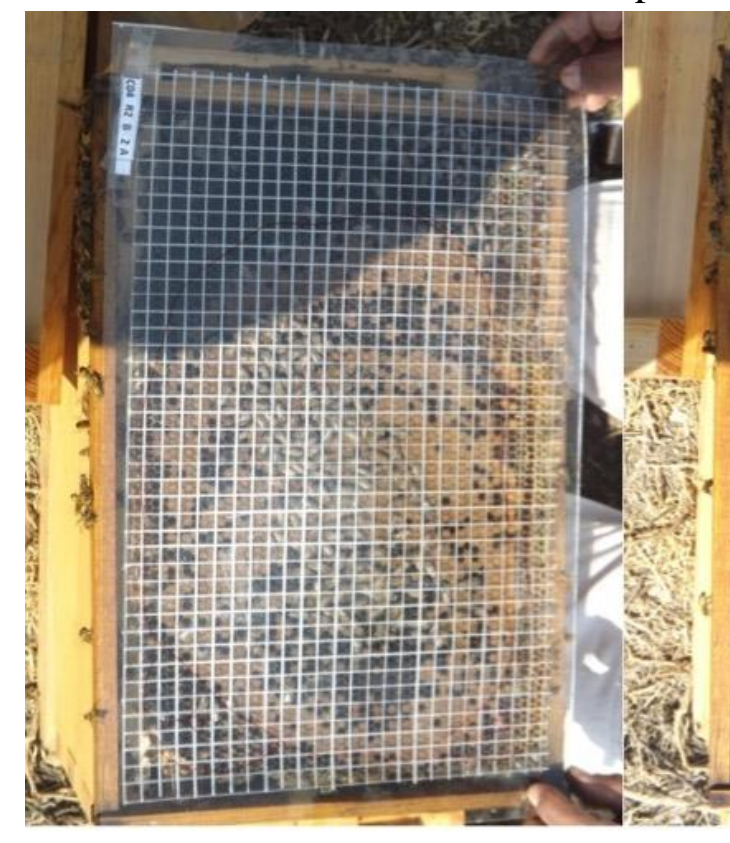

A

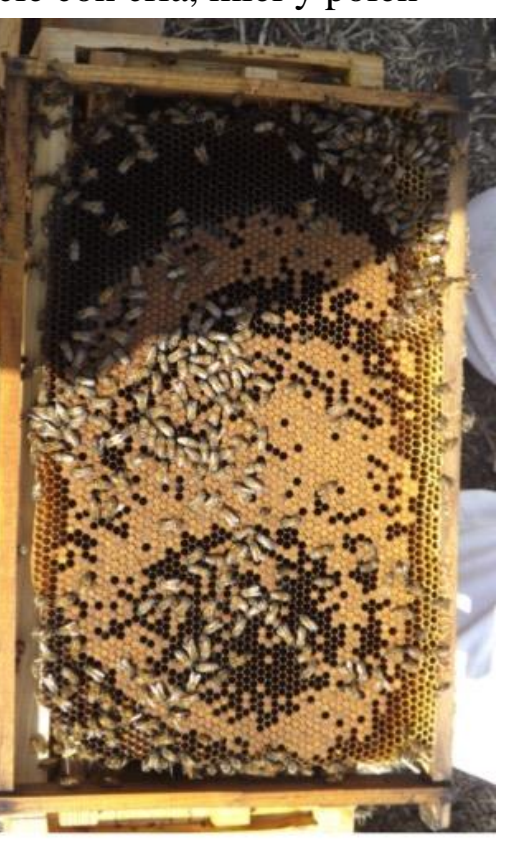

B

\section{Análisis estadístico}

Se utilizó un análisis de varianza de una vía (ANOVA) para determinar si existen diferencias significativas entre los tratamientos (tipos de colmena) en el área de cría, miel y polen para cada fecha de muestreo. Las temperaturas promedio en el centro y a la altura del cabezal a lo largo del experimento se compararon también con un ANOVA. Se verificaron los supuestos del ANOVA utilizando la prueba de Kolmogorov-Smirnov para la normalidad y la prueba de Levene para la homogeneidad de varianzas y en caso de no cumplirse, se utilizó un ANOVA no paramétrico (Kruskal-Wallis). Se aplicó la prueba de comparación múltiple de Tukey en los casos en los que se obtuvieron diferencias 
significativas $(P<0.05)$. Los análisis estadísticos se realizaron con el paquete estadístico SigmaStat $3.5^{(27)}$. Se reportan promedios \pm error estándar.

\section{Resultados}

La superficie con cría de A. mellifera en la primera fecha de muestreo ( 2 de febrero) fue de $1,125.73 \pm 136.65 \mathrm{~cm}^{2}$ para las colmenas de $23.9 \mathrm{~L}, 1,016.75 \pm 364.64 \mathrm{~cm}^{2}$ para las de 42.3 $\mathrm{L}$ y 1,398.63 $\pm 334.67 \mathrm{~cm}^{2}$ para las de $52.2 \mathrm{~L}$ y estos valores no difirieron significativamente entre sí $(F=0.44, g . l .=2,11, P>0.05)$ (Figura 3). La superficie con cría de A. mellifera en la segunda fecha de muestreo ( 7 de marzo) fue de $1,610.25 \pm 83.37 \mathrm{~cm}^{2}$ para las colmenas de $23.9 \mathrm{~L}, 1,654.75 \pm 473.37 \mathrm{~cm}^{2}$ para las de $42.3 \mathrm{~L}$ y 1,692.75 \pm 68.03 $\mathrm{cm}^{2}$ para las de $52.2 \mathrm{~L}$ y estos valores no difirieron significativamente entre sí $(H=0.50$, g.l. $=2, P>0.05)$. La superficie con cría sí difirió significativamente entre tipos de colmena al final del experimento ( 28 de abril) $(F=23.88$, g.l. $=2,11, P<0.001)$. La superficie con cría en colmenas con un volumen interno de $23.9 \mathrm{~L}$ fue en promedio de $3,077.25 \pm 81.81 \mathrm{~cm}^{2} \mathrm{y}$ en las colmenas de $42.3 \mathrm{~L}$ fue de 2,906.2 $\pm 94.6 \mathrm{~cm}^{2}$ y ambos valores fueron significativamente mayores que el valor en las colonias que se desarrollaron en colmenas de 52.2 L, cuya superficie con cría fue de 2,331.2 $\pm 59.5 \mathrm{~cm}^{2}(P<0.01)$. La superficie con cría en las colmenas de 23.9 L no difirió significativamente de aquella en las colmenas de $42.3 \mathrm{~L}(P>0.05)$.

Figura 3: Superficie con cría (promedio \pm error estándar) en tres tamaños de colmenas al final del periodo de estudio

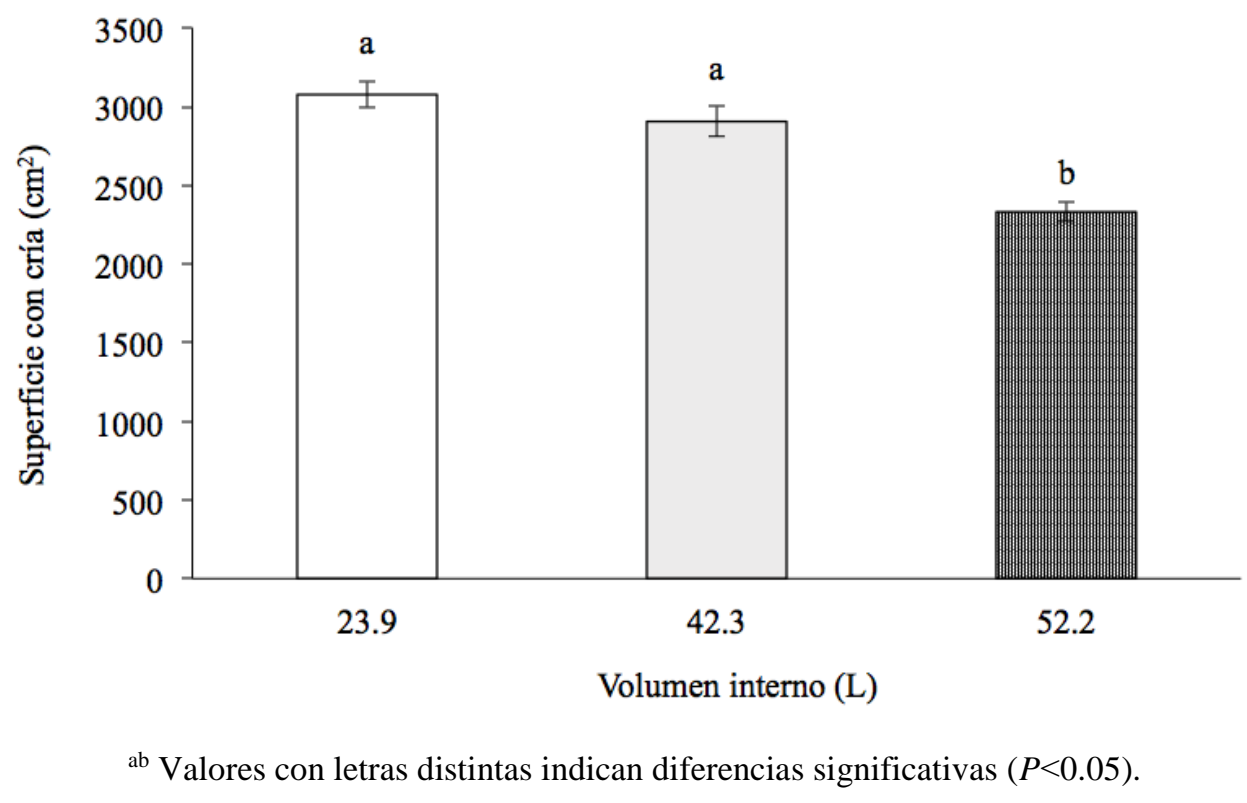


La superficie con miel en la primera fecha de muestreo fue de $980.38 \pm 263.64 \mathrm{~cm}^{2}$ para las colmenas de $23.9 \mathrm{~L}$, de $952.75 \pm 201.76 \mathrm{~cm}^{2}$ para las de $42.3 \mathrm{~L}$ y $992.50 \pm 93.93 \mathrm{~cm}^{2}$ para las de 52.2 L ( $F=0.01$, g.l. $=2,11, P>0.05$ ) (Figura 4). La superficie con miel en la segunda fecha de muestreo fue de $905.75 \pm 198.38 \mathrm{~cm}^{2}$ para las colmenas de $23.9 \mathrm{~L}, 465.25 \pm$ $167.03 \mathrm{~cm}^{2}$ para las de $42.3 \mathrm{~L}$ y $621.50 \pm 184.88 \mathrm{~cm}^{2}$ para las de $52.2 \mathrm{~L}(F=1.48$, g.l.=2,11, $P>0.05)$. La superficie con miel al final del periodo de estudio sí difirió significativamente entre tipos de colmena $(H=8.80$, g.l. $=2,11, P<0.01)$. El promedio de la superficie con miel en las colmenas de $23.9 \mathrm{~L}\left(1424 \pm 56.9 \mathrm{~cm}^{2}\right)$ fue significativamente mayor que en las colmenas de $52.2 \mathrm{~L}\left(849.5 \pm 94.4 \mathrm{~cm}^{2}, P<0.05\right)$, pero no difirió significativamente del valor en las colonias de $42.3 \mathrm{~L}(1056 \pm 19.7, P>0.05)$. La superficie con miel en las colmenas de 42.3 y $52.2 \mathrm{~L}$ fue similar $(P>0.05)$.

Figura 4: Superficie con miel (promedio \pm error estándar) en tres tamaños de colmenas al final del periodo de estudio

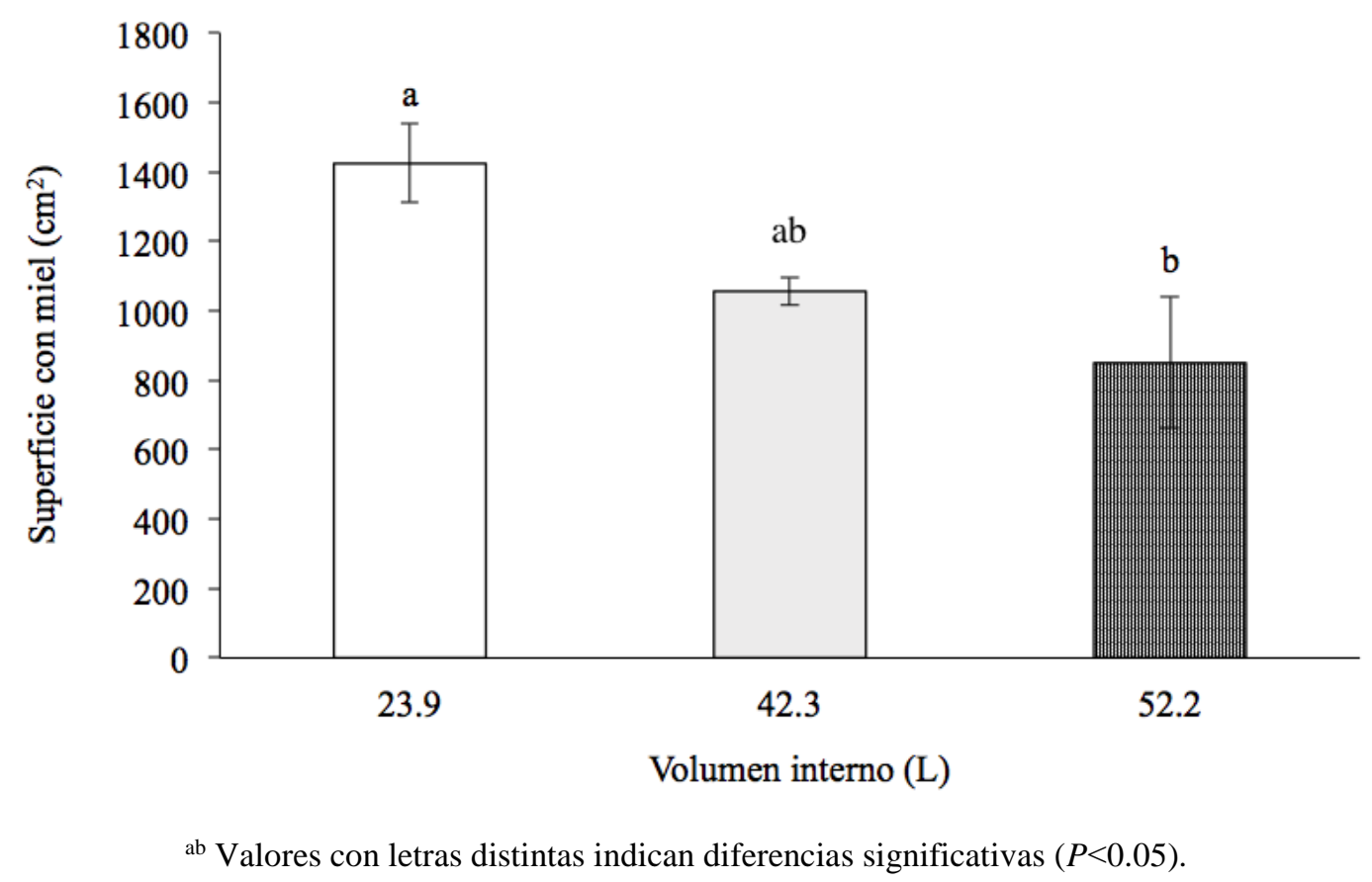

La superficie con polen en la primera fecha de muestreo fue de $136.38 \pm 26.67 \mathrm{~cm}^{2}$ para las colmenas de $23.9 \mathrm{~L}$, de $242.75 \pm 147.59 \mathrm{~cm}^{2}$ para las de $42.3 \mathrm{~L}$ y $112.50 \pm 13.14 \mathrm{~cm}^{2}$ para las de $52.2 \mathrm{~L}(H=0.34$, g.l. $=2, P>0.05)$. La superficie con polen en la segunda fecha de muestreo fue $252.75 \pm 77.03 \mathrm{~cm}^{2}$ para las colmenas de $23.9 \mathrm{~L}$, de $146.5 \pm 35.11 \mathrm{~cm}^{2}$ para las de $42.3 \mathrm{~L}$ y $192.5 \pm 43.19 \mathrm{~cm}^{2}$ para las de $52.2 \mathrm{~L}(F=0.94$, g.l. $=2,11, P>0.05)$. La superficie con polen al final del periodo de estudio sí difirió significativamente entre tipos de colmena $(F=9.12$, g.l. $=2,11, P<0.01)$ (Figura 5). La superficie con polen al final del periodo de estudio fue significativamente mayor en la colmena de $23.9 \mathrm{~L}(227.0 \pm 37.0$ 
$\left.\mathrm{cm}^{2}\right)$ que en las colmenas de $42.3 \mathrm{~L}\left(87.7 \pm 29.9 \mathrm{~cm}^{2}\right)$ y $52.2 \mathrm{~L}\left(56.2 \pm 21.3 \mathrm{~cm}^{2}\right)(P<0.05)$. La superficie con polen en las colmenas de 42.3 y $52.2 \mathrm{~L}$ no difirió significativamente $(P>0.05)$ (Figura 5).

Figura 5: Superficie con polen (promedio \pm error estándar) en tres tamaños de colmenas al final del periodo de estudio

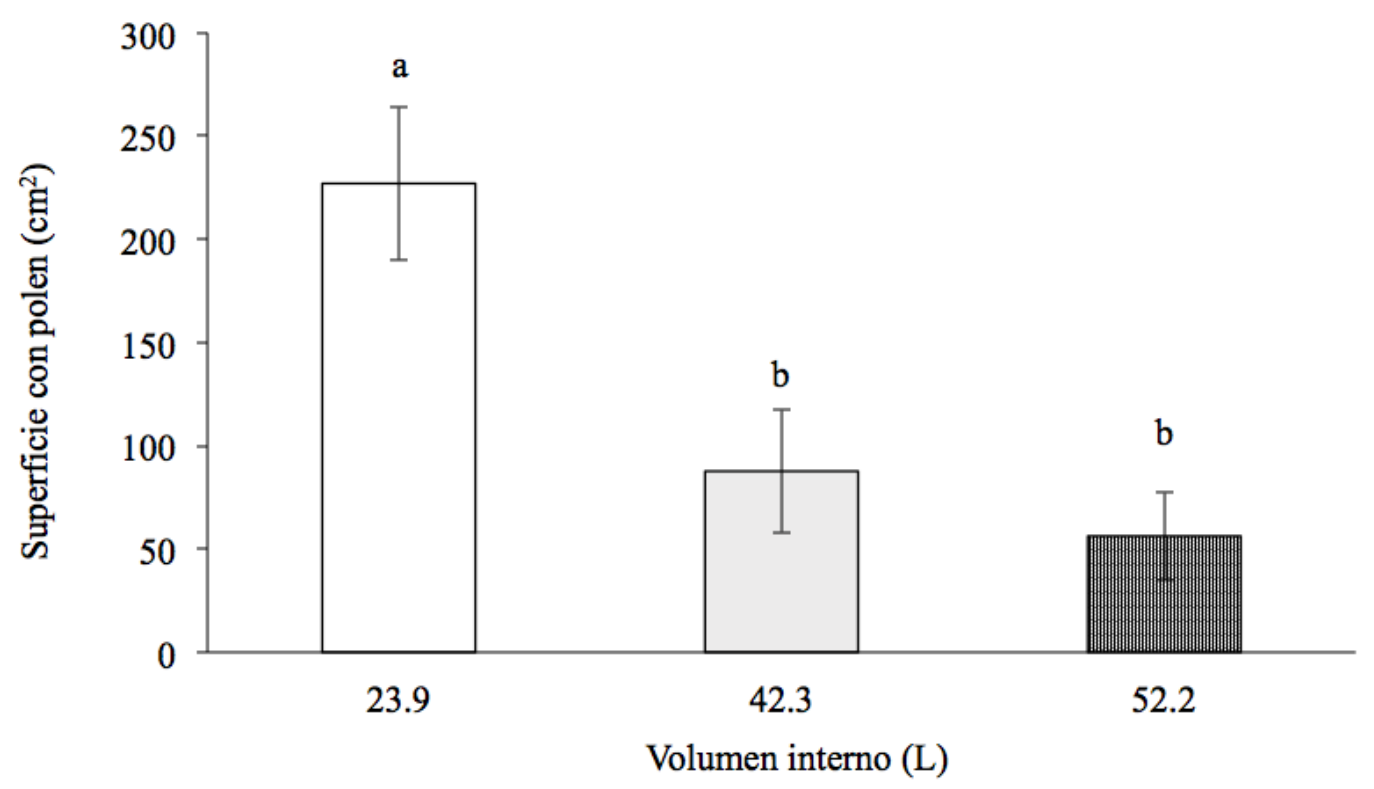

${ }^{\text {ab }}$ Valores con letras distintas indican diferencias significativas $(P<0.05)$.

La temperatura promedio en el centro de las colmenas fue relativamente estable a lo largo del día durante el experimento, a pesar de que la temperatura ambiental varió en promedio entre 7 y $33{ }^{\circ} \mathrm{C}$ (Figura 6). La temperatura en el centro de las colmenas de $23.9 \mathrm{~L}$ fue de $33.6 \pm 1.0{ }^{\circ} \mathrm{C}$, en las de $42.3 \mathrm{~L}$ fue de $33.4 \pm 0.8^{\circ} \mathrm{C}$ y en las de $52.2 \mathrm{~L}$ fue de $33.7 \pm 0.9{ }^{\circ} \mathrm{C}$ $(F=0.04, g . l .=2,11, P>0.05)$. La temperatura promedio a la altura del cabezal varió a lo largo del día en los tres tipos de colmenas y fue significativamente mayor en las colmenas de $23.9 \mathrm{~L}\left(23.4 \pm 0.5^{\circ} \mathrm{C}\right)$ y de $42.3 \mathrm{~L}\left(23.8 \pm 0.6^{\circ} \mathrm{C}\right)$ que en las colmenas de $52.2 \mathrm{~L}(21.4 \pm$ $\left.0.4{ }^{\circ} \mathrm{C}\right)(F=6.92$, g.l. $=2,11, P<0.05)$ (Figura 7). La temperatura a la altura del cabezal en las colmenas de 23.9 y $42.3 \mathrm{~L}$ no difirió significativamente $(P>0.05)$. 
Figura 6: Temperatura ambiental y en el interior (centro y cabezal del tercer bastidor) en tres tamaños de colmenas

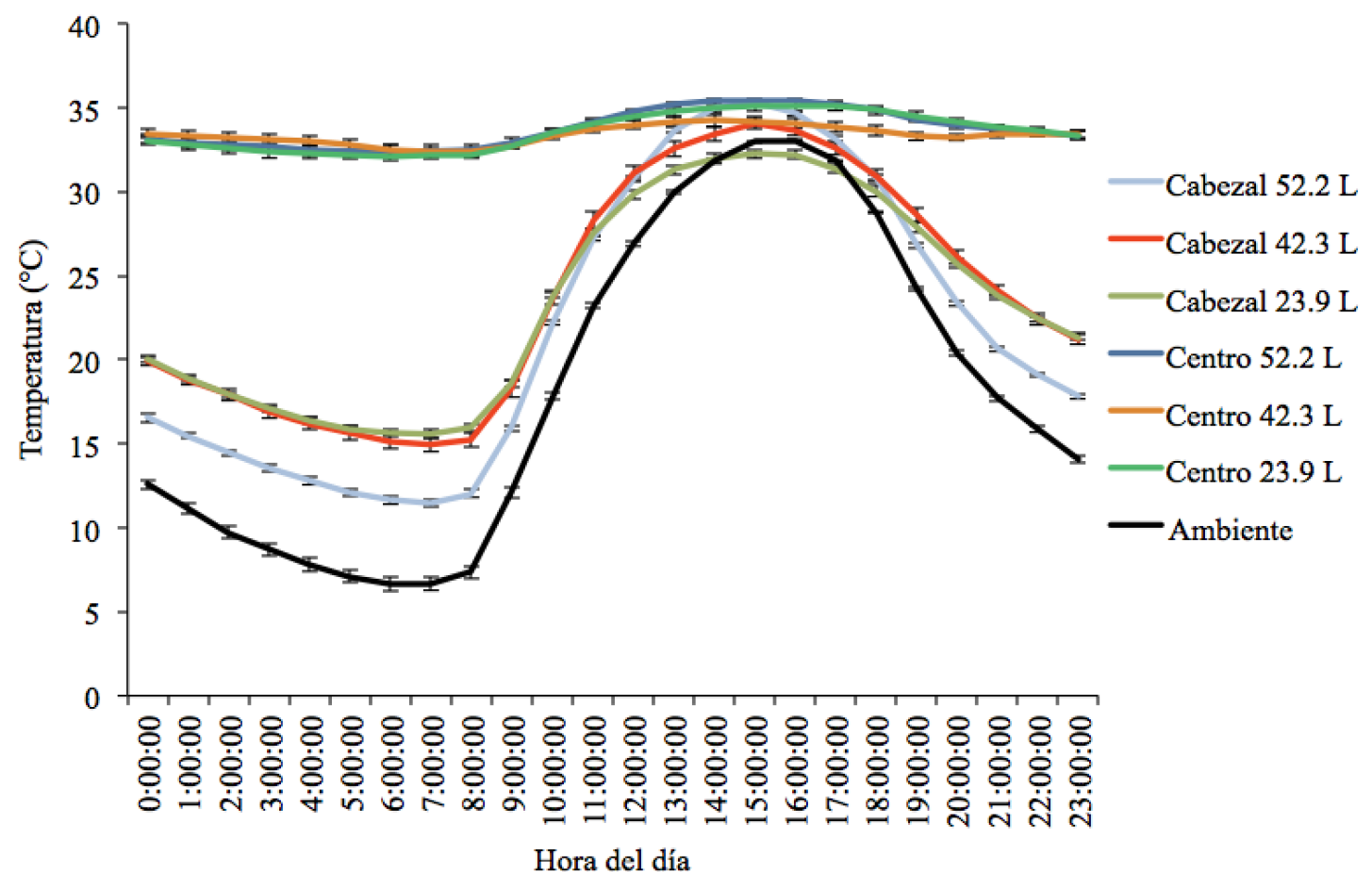

Los valores representan promedios \pm error estándar.

Figura 7: Temperatura a la altura del cabezal (promedio \pm error estándar) en tres tamaños de colmenas durante el periodo de estudio

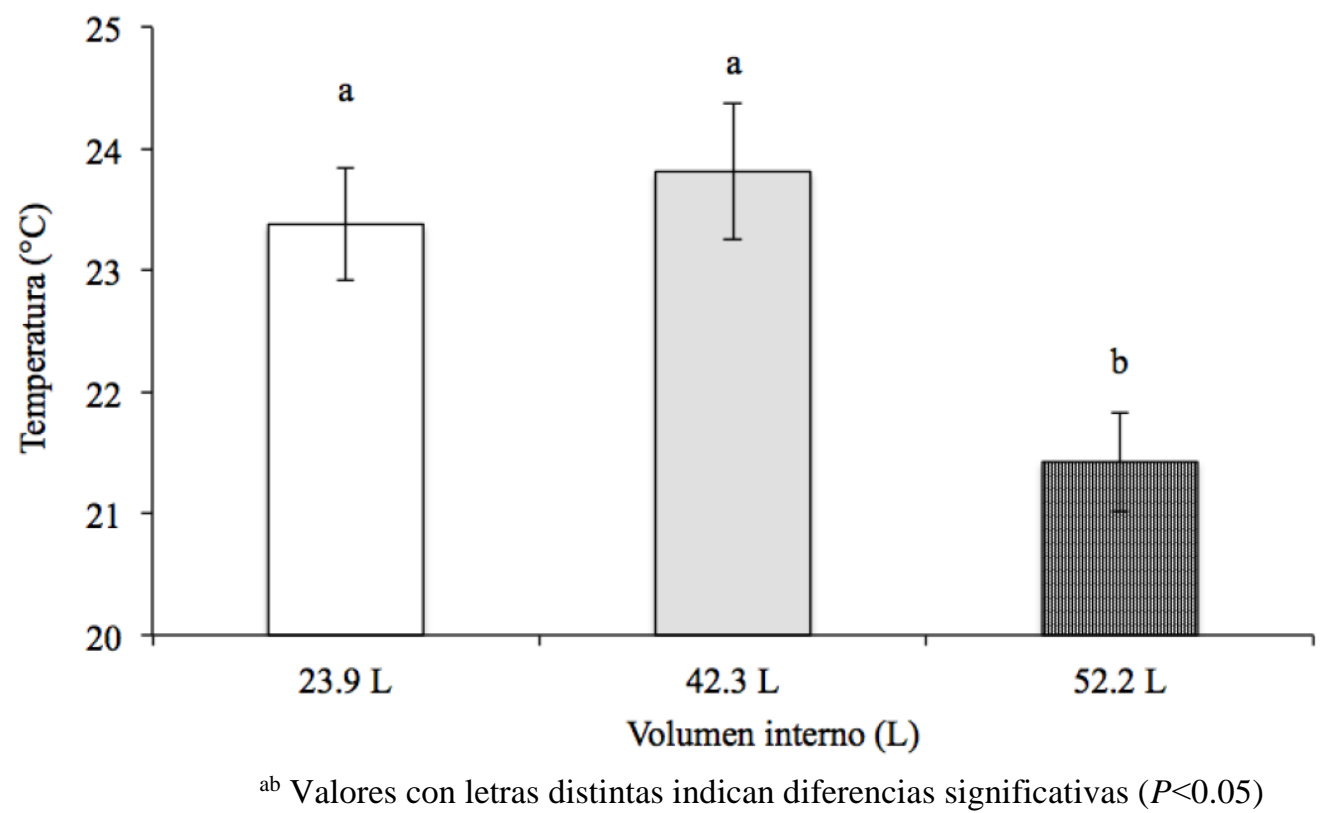




\section{Discusión}

Los resultados obtenidos en este trabajo muestran que el desarrollo de la colonia expresado en superficie con cría al final del experimento fue menor cuando éstas se encontraban en las cámaras de cría tipo Jumbo, las de mayor tamaño (Figura 3). De manera similar, la superficie con miel y polen fue menor en las colmenas con volúmenes internos de mayor tamaño (52.2 y 42.3 L) que en las colmenas experimentales de $23.9 \mathrm{~L}$ (Figuras 4,5). Estos resultados concuerdan con los reportados por Abd-Elmawgood et al $l^{(15)}$ quienes compararon tres tamaños internos de colmenas $(38,31$ y 24 L) y la mejor respuesta (mayor cantidad de cría, polen y miel) la obtuvieron con las colmenas de menor tamaño. Al igual que en nuestro trabajo, también encontraron que las diferencias en cantidad de cría, polen y miel entre colmenas de diferentes tamaños internos se manifestaron al final del invierno e inicio de la primavera. De manera similar, Ballesteros et $a l^{(28)}$ reportaron que la producción de jalea real fue mayor en colmenas de recría de 6 bastidores que en colmenas de 8 y 10 bastidores.

También se encontró que la temperatura en el centro del nido de cría de A. mellifera no difirió significativamente con respecto al tamaño de la colmena, lo cual indica que independientemente del tamaño de la colmena, las abejas pueden termorregular y mantener la cría a temperaturas alrededor de $33{ }^{\circ} \mathrm{C}$ para que su progenie se desarrolle adecuadamente ${ }^{(7,8)}$. Sin embargo, la temperatura en el cabezal fue significativamente menor en las colmenas tipo Jumbo (52.2 L) que en las de 42.3 y 23.9 L (Figuras 6,7). Estos resultados muestran que en las cámaras de cría de menor tamaño las abejas pueden conservar de forma más eficiente el calor generado durante el calentamiento del nido de cría, lo cual ya había sido sugerido ${ }^{(15,28)}$.

El menor porcentaje de superficie con cría, miel y polen encontrado en las colmenas de mayor tamaño puede estar relacionado con diferentes factores. Es probable que las abejas hayan consumido más miel en las colmenas con menor temperatura interna para obtener la energía metabólica necesaria para termorregular ${ }^{(29,30)}$. Aunque no se cuantificó directamente la producción de calor por las abejas, sí se observó que en las colmenas tipo Jumbo las obreras permanecían agregadas alrededor del nido de cría durante más tiempo que en las colmenas de menor tamaño, lo cual sugiere que las obreras en las colmenas de mayor tamaño invirtieron más tiempo en la termoregulación que en la producción de cría ${ }^{(31)}$ o en el pecoreo ${ }^{(2,3,18)}$. Adicionalmente, una mayor temperatura dentro de las colmenas de menor tamaño pudo facilitar la construcción del nido ya que la elasticidad de la cera aumenta y el gasto energético para moldearla disminuye con el incremento de la temperatura $^{(32,33)}$. Finalmente, es necesario considerar que el desarrollo de la colonia también depende de los adultos presentes durante la época invernal ${ }^{(17)}$, sin embargo, en este trabajo no se cuantificaron para evitar que su manipulación afectara la cohesión social de 
las colonias ${ }^{(24)}$, pero es necesario que estos datos sean considerados en estudios posteriores.

Se ha planteado que es necesario utilizar colmenas que permitan incrementar la temperatura interna de la colmena de la abeja europea durante la temporada invernal ${ }^{(34,35,36)}$. En este trabajo se compararon dos tipos de colmenas utilizadas comúnmente en México (Jumbo y Langstroth) y una colmena experimental de menor tamaño. Los resultados muestran que la colmena experimental provee de mejores condiciones térmicas y de un aumento en la fortaleza (mayor cantidad de cría) y productividad de la abeja melífera. Sin embargo, los requerimientos térmicos y de espacio interno de la colmena de A. mellifera pueden variar entre razas y entre climas ${ }^{(37-40)}$, por lo que es necesario realizar estudios bajo las condiciones específicas de cada región apícola.

\section{Conclusiones e implicaciones}

La mejor respuesta de fortaleza (mayor cantidad de cría) y mayores reservas de miel de la abeja melífera se registró cuando se utilizó la colmena con el menor tamaño interno (23.9 L). La colmena tipo Jumbo, utilizada en el altiplano central de México, presentó los valores más bajos de miel, polen y cría al final de la temporada invernal. Esto puede repercutir en una disminución de la productividad de miel y polen para los apicultores que utilizan este tipo de colmena, por lo que es conveniente implementar alguna práctica o mecanismo que permita mantener las colonias de abejas fuertes para la cosecha de primavera. Por ejemplo, se puede alimentar a las abejas con agua y sustitutos de néctar y polen y disminuir el tamaño interno de la colmena utilizando reductores de espacio. La época de invierno tradicionalmente es considerada una temporada donde la abeja reina deja de poner huevos y los requerimientos térmicos y alimenticios de la colonia disminuyen. Sin embargo, durante el trabajo realizado en el municipio de Tizayuca se registraron temperaturas ambientales superiores a $24{ }^{\circ} \mathrm{C}$ por más de $6 \mathrm{~h}$ al día durante el invierno, lo que permitió que la abeja reina pudiera mantener la postura de huevos durante esta época. Este comportamiento tan diferente hace necesario un mejor seguimiento del apicultor para mantener las colonias de abejas con suficientes reservas de alimento durante el invierno para evitar que se debiliten.

\section{Agradecimientos}

Al Consejo Nacional de Ciencia y Tecnología (CONACYT) por la beca otorgada a AHC durante la realización de este trabajo. También se agradece al Centro de Investigaciones Biológicas y al Programa Anual de Investigación (2016) de la Universidad Autónoma del Estado de Hidalgo, así como a REFAMA (REFAMA CONACYT clave 251272 "Red Temática Biología, Manejo y Conservación de Fauna Nativa en Ambientes Antropizados" por el apoyo otorgado durante la realización de este trabajo. Finalmente, agradecemos a Iriana Zuria y a dos revisores anónimos su valiosa ayuda para mejorar el manuscrito. 


\section{Literatura citada:}

1. Jones CV, Oldroyd BP. Nest thermoregulation in social insects. Adv Insect Physiol 2007;33:153-171.

2. Burril RM, Dietz A. The response of honey bees to variations in solar radiation and temperature. Apidologie 1981;12(4):319-328.

3. Reyes CJL, Cano RP. Manual de polinización apícola. Programa nacional para el control de la abeja africana-Instituto interamericano para la cooperación agrícola. Manual no. 7. Distrito Federal, México: Secretaría de Agricultura, Ganadería, Pesca y Alimentación (SAGARPA); 2003.

4. Dunham WE. Temperature gradient in the egg-laying activities of the queen bee. Ohio J Sci 1930;30(6):403-410.

5. Yuca-Rivas R. Variación intranual en el espectro polínico de la miel producida en Huarán (Cusco, Perú). Ecol Apl 2016;15(1):27-36.

6. Cho LH, Gynheung A. The control of flowering time by environmental factors. Plant $\mathbf{J}$ 2017;90:708-719.

7. Heinrich B. The hot-blooded insects: strategies and mechanisms of thermoregulation. Alemania, Berlin: Springer-Verlag; 1993.

8. Winston ML. The biology of the honey bee. Cambridge, Massachusetts, EUA: Harvard University Press; 1987.

9. Seeley TD, Morse RA. The nest of the honey bee (Apis mellifera L.). Insectes Soc 1976;23(4):495-512.

10. Seeley TD, Morse RA. Nest site selection by the honey bee, Apis mellifera. Insectes Soc 1978;25(4):323-337.

11. Seeley TD. Measurement of nest-cavity volume by the honey bee (Apis mellifera). Behav Ecol Sociobiol 1977;2(2):201-227.

12. Szabo TI. The Thermology of wintering honeybee colonies in 4-colony packs as affected by various hive entrances. J Apic Res 1985;24(1):27-37.

13. Toomemaa K, Mand M, Williams IH. Wintering of honey bee colonies in cylindrical nest cavities versus oblong box-hives in a North European climate. J Apic Res 2016;54(4):105-111. 
14. Ros PJM. Iniciación a la apicultura. Murcia, España: Comunidad Autónoma de la Región de Murcia; 2009.

15. Romero NJM. Diseño de colmena [tesis maestría]. Ciudad de México, México; Universidad Nacional Autónoma de México; 2017.

16. SAGARPA. Manual de buenas prácticas pecuarias en la producción de miel. Distrito Federal, México: Secretaría de Agricultura, Ganadería, Desarrollo Rural, Pesca y Alimentación (SAGARPA);2010.

17. Jean-Prost P, Le Conte Y. Apicultura: conocimiento de la abeja, manejo de la colmena $4^{\mathrm{a}}$ ed. Barcelona, España: Mundi-Prensa; 2006.

18. Abd-Elmawgood BH, Al-Rajhi MA, El-Ashhab AO. Effect of the internal size and thermal insulation of the hive on bee colonies strength and productivity. Egyptian J Agric Res 2015;93:185-195.

19. Hoover SER, Hoover TM. Impact of environmental change on honeybees and beekeeping. In: Gupta R, et al editors. Beekeeping for poverty alleviation and livelihood security. Nueva York, EUA: Springer; 2014:463-480.

20. Reddy PVR, Verghese A, Rajan VV, Potential impact of climate change on honeybees (Apis spp.) and their pollination services. Pest Manag Hortic Ecosyst 2012;18:121-127.

21. Contreras EF, Pérez AB, Echazarreta CM, Cavazos AJ, Macías MJO, Tapia GJM. Características y situación actual de la apicultura en las regiones Sur y Sureste de Jalisco, México. Rev Mex Cienc Pecu 2013;4(3):387-398.

22. SPDRMH. Secretaría de planeación y desarrollo regional y metropolitano del estado de Hidalgo, México. http://intranet.ehidalgo.gob.mx/NormatecaE/Archivos/archivo6405.pdf. Consultado 30 Ago, 2017.

23. DCM. Datos Climáticos Mundiales. https://es.climate-data.org/. Consultado 4 Jun, 2017.

24. Delaplane KS, Steen JVD, Guzman-Novoa E. Standart methods for estimating strength parameters on Apis mellifera colonies. J Apic Res 2013;52(1):1-12.

25. Cervantes GER. Incidencia de la alimentación suplementaria en la producción y productividad de la apicultura (Apis mellifera) [tesis licenciatura]. Colimbuela, Ecuador; Universidad Técnica del Norte; 2010.

26. Martínez GEG, Pérez LH. La producción de miel en el trópico húmedo de México: avances y retos en la gestión de la innovación $1^{\text {a }}$ ed. Texcoco, México: Universidad Autónoma Chapingo; 2013. 
27. Systat Software. SigmaStat 3.5. Chicago, EUA: Systat Software; 2006.

28. Ballesteros HH, Vásquez RE. Determinación de jalea real en colmenas de recría de diferentes dimensiones. Revista Corpoica 2007;8(1):75-78.

29. Southwick E. Metabolic energy of intact honey bee colonies. Com Biochem Physiol 1982;71(2):277-281.

30. Seeley TD, Visscher PK. Survival of honeybees in cold climates: the critical timing of colony growth and reproduction. Ecol Entomol 1985;10(1)81-88.

31. Vogt FD. Thermoregulation in bumblebee colonies. I. Thermoregulatory versus broodmaintenance behaviors during acute changes in ambient temperature. Physiol Zool 1986;59(1):55-59.

32. Hepburn HR. Honeybees and wax, an experimental natural history. Heidelberg, Alemania: Springer; 1986.

33. Karihaloo BL, Zhang K, Wang J. Honeybee combs: how the circular cells transform into rounded hexagons. J R Soc Interface 2013;10(86):20130299.

34. Abou-Shaara HF, Oways AA, Ibrahim YY, Basuny NK. A review of impacts of temperature and relative humidity on various activities of honey bees. Insectes Soc 2017;64(4):455-462.

35. Wineman E, Lensky Y, Mahrer Y. Solar heating of honey bee colonies (Apis mellifera L.) during the subtropical winter and its impact on hive temperature, worker population and honey production. Am Bee J 2003;43(7):565-570.

36. Erdogan Y, Dodologlu A, Emsen B. Some physiological characteristics of honeybee (Apis mellifera L.). housed in heated, fan wooden and insulated beehives. J Anim Vet Adv 2009;8(8):1516-1519.

37. Gould JL. Why do honey bees have dialects? Behav Ecol Sociobiol 1982;10(1):53-56.

38. Schmidt JO, Hurley R. Selection of nest cavities by Africanized and European honey bees. Apidologie 1995;26(6):467-475.

39. Schneider S, Blyther R. The habitat and nesting biology of the African honey bee Apis mellifera scutellata in the Okavango River Delta, Botswana, Africa. Insectes Soc 1988;35(2):167-181.

40. Hoover SER, Hoover TM. Beehives in the world. In: Gupta R, Reybroeck W, van Veen J, Gupta A editors. Beekeeping for poverty alleviation and livelihood security. Nueva York, EUA: Springer; 2014:125-170. 